\title{
Identifying Critical Success Factors of Financial ERP System in Higher Education Institution using ADVIAN® Method
}

\author{
Ayogeboh Epizitone ${ }^{1}$, Oludayo. O. Olugbara ${ }^{2}$ \\ ICT and Society Research Group, South Africa Luban Workshop \\ Durban University of Technology \\ Durban, South Africa
}

\begin{abstract}
Enterprise resource planning (ERP) has been widely accepted by many organizations as an information technology process to seamlessly integrate, manage, and boost performance in different units of an organization. However, there linger an unpleasant chasm on success and satisfaction rates of ERP system implementation that have limited the effective use of the system. Moreover, the critical success factors (CSFs) of ERP system implementation have not been investigated in the literature for the case of financial functions in higher education institutions. This paper, through the application of advanced impact analysis (ADVIAN $®$ ) method exploits the CSFs of ERP system to support financial functions in a higher education institution. The applied ADVIAN $₫$ method highlights the CSFs that are measured according to the measures of criticality, integration, and stability. Furthermore, using precarious, driving, and driven measurements for ranking the factors, an effective model of CSFs for a financial ERP system implementation is attained to support financial functions. The study findings provide a comprehensive methodological scheme that can be used as a reference guide and as an orientation point for efficacious planning, implementing, and using ERP systems to support financial functions in higher education institutions.
\end{abstract}

Keywords - Cross impact; enterprise resource; impact analysis; resource planning; success factor

\section{INTRODUCTION}

Enterprise resource planning (ERP) system implementation has been contextualized in past research studies as a medium for modernization and reformation of many organizations [1, 2]. The impetus for the adoption of ERP systems has been evident in literature for reasons such as pressure of competitiveness and improvement in operational efficiency [1, 3]. Many definitions exist in literature which describe ERP system as a unified software interface application with one large database, software package to facilitate seamless integration, configurable information system packages and computer-based systems [4, 5]. Al-Hadi and Al-Shaibany [4] consolidated diverse definitions of an ERP system as a software, a concept, a system or a package that integrates multiple modules as a separate functional area that includes a set of business processes with data flowing into a central database that could be uploaded locally or into the cloud. In the context of higher education institutions (HEIs), an ERP system has been defined as an information technology application that integrates automated recruitment, student admission, financial aid, student records, and many academic and administrative services $[2,6]$.

Notwithstanding of the diverse definitions, an ERP system can be implemented in any organization regardless of size or nature [4]. Literature highlights the notable increase in an ERP system implementation and utilization from its onset till the present [3, 6]. However, this endeavor has been considered problematic, especially in HEIs that implement ERP systems to enhance quality of academic and administrative services [3, $6,7]$. Previous authors have attributed the causes of these snags to the lack of contextualization and deficiency of specific knowledge required [1, 3, 8]. Furthermore, authors have highlighted the existence of a solemn literature chasm that submerges the implementation of ERP systems in developing countries [9], and insufficient research conducted on successful implementations of ERP systems in HEIs [8]. Hence, with these chasms in mind, this paper seeks to make a significant contribution to the ERP phenomenon using a rigorous scientific method that will benefit practitioners and researchers.

The objective of this study was to investigate the critical success factors (CSFs) of ERP system implementation for the case of financial functions in higher education institutions using advanced impact analysis (ADVIAN@) method. Financial function is the business process of planning, acquiring, controlling, managing, and utilizing funds for the effective operations of an organization. The content of this paper is concisely structured as follows. Section 1 presents the introductory message. Section 2 reviews the related literature while Section 3 describes the ADVIAN@ method. Section 4 presents the results of the analysis. Section 5 discusses the results and the paper is succinctly concluded in Section 6.

\section{LITERATURE REVIEW}

Large scale fragmentation in HEIs has caused many of the institutions to seek for a unified ERP platform that can seamlessly integrate disparate information systems [1]. However, the implementation of ERP systems has been flouted with many challenges as previously mentioned. In the case of financial systems, there are reports of ERP systems lagging in real-time and not being supportive of the major financial functions in HEIs $[10,11]$. The modular design of an ERP system caters for different organizational processes, but 
each module that is not financially inclined correlates regularly with the financial module. In a complex interconnected system, a non-financial module provides and concomitantly needs useful information from the financial module for aspects such as reporting, budgeting, salaries, and fees. This concern was echoed by Noaman and Ahmed [11], that highlighted the deficiency of vendor proprietary ERP systems which need to response to the real functionalities of HEIs.

There have been red flags of the ERP systems not meeting the functional needs of HEIs despite that the space is progressing rapidly $[11,12]$. Despite being one of the most frequently used systems [6], there is an alarming high rate of failure stemming from the implementation of ERP systems in HEIs [5]. Different authors have posited the implementation of ERP systems in HEIs to be complicated and presenting risks with no factors that can guarantee a successful implementation [4-6]. The plethora of intrinsic challenges of ERP systems posit the inadequacy of quality research that consider the uniqueness of the functional needs of HEIs [3, 5].

Previous studies have attempted to fill these chasms by proposing the identification of CSFs for implementation of ERP systems that targeted principal areas of business operations to ensure success. However, there has been censure for the inadequacy of sound scientific methods that can provide robust empirical evidence to support research findings $[5,8]$. The concept of CSFs has been well research in many organizational environments, but little contributions have been experienced in the context of HEIs. Higher education system is attracting the keen interest of software vendors who view the system as a lucrative industry that is worth several hundred of billion dollars in revenue [11]. Several studies have delved into this dimension to derive CSFs for implementation of ERP systems in HEIs [5].

There have been a plethora of extensive studies on ERP implementation over the past decades that have contributed significantly to the understanding of the concept of CSFs [13]. However, within the extant literature, the identification of CSFs still require further investigation with authors calling for the application of more rigorous scientific methods [5, 8]. As a result, many of these authors have responded to the call by adopting CSFs to highlight areas that are critical for the successful implementation of ERP systems [13]. It was alluded by Loonam, Kumar, Mitra and Abd Razak [13] that CSFs are a powerful enabler that are extensively recognized within the ERP literature for identifying germane issues that require organizational attention before and during an ERP system implementation.

Shatat [14] has emphasized the importance of identifying CSFs of implementing ERP systems to facilitate the continuous success of the system and guarantee an improved influence on business performance. Sowan, Tahboub and Khamayseh [6] concentrated on technical success factors of an ERP system implementation. They identified 10 CSFs indicating the importance of factors that could support the structure that needs to be followed during implementation. Soliman and Karia [15] discussed a relatively small number of CSFs for implementing ERP systems in HEIs. They highlighted the importance of CSFs while providing better understanding of whether their role is limited to influencing results at relevant stages in an innovation process. In addition, authors have highlighted the need for CSFs to maximize the potential outcome of an ERP system implementation with literature evidence to serve as footing to derive the factors using a sound scientific method that satisfies the need for analytically derived factors [16].

\section{METHOD OF ADVIAN®}

This study follows a series of stages to identify CSFs that were subjected to expert evaluation which has led to the determination of cross-impact matrix and subsequent application of ADVIAN®, which is discussed briefly in this section. The ADVIAN® ${ }^{\circ}[17,18]$ has been used in this study to determine the impacts that various CSFs have on a financial ERP system. This will allow cross-impact analysis that offers a provision for organizations to explore the current challenges and adequately prepare the right decisions for future endeavors in a manner that is participatory $[5,8,17]$. Impact analysis has been extensively employed in previous times in scenario analysis and future undertakings [17, 19]. It is currently employed in performance measurement to map tangible and intangible relationships [5, 17, 19].

The cross-impact analysis method utilizes an impact matrix that is filled based on the impact strength of the factors concerned. There are various measures of impact strength that have been used such as a rating score from 0 to 3 , where 0 signifies no impact, 1 is a weak impact, 2 is a medium impact and 3 indicates a strong impact $[5,17]$. This study rather uses a normalized cross-impact matrix with the impact strength normalized in the interval of 0 to 1 [5]. In this normalized range, 0.00 to 0.25 signifies no impact, 0.26 to 0.50 is a weak impact, 0.51 to 0.75 is a medium impact and 0.76 to 1.00 signifies a strong impact. Early cross-impact analysis methods did not have the ability of analyzing indirect interrelationships that gave rise to the alternate. Matrice d'Impacts Croisés Multiplication Appliquée à un Classement (MICMAC) addressed the inadequacy of direct impact [19]. However, this method along with other cross-impact analysis methods such as Papier computer and Fuzzy approach present intrinsic deficiencies because they consider either direct or indirect interrelations and fail to deal with both aspects concomitantly [5, 17-20].

The ADVIAN $®$ is an improved quantitative impact analysis method that considers the indirect interactions amongst factors in a more reliable manner. The method uses active sum, passive sum and the impact strength of the corresponding factor for determining indirect interdependencies. Base on the method, CSFs can be identified reliably. However, it is limited because it does not measure the conditions of impact factors, but their interactions [18]. Hence, ADVIAN® does not give the status of a system, but rather identifies the important factors necessary for the entire system performance and regulation that justifies its application in this study. Additionally, ADVIAN@ is favored over other methods for reasons such as the ability to provide deeper insights where other methods have proven inadequate and to provide an understanding of mutual relationship 
between two single resources $[5,17,18,20]$. Furthermore, it is suitable for explorative modeling as it overcomes the impossibility of privation of theory-based computational models that are because of inadequate theoretical advancement, establishing interrelationships and mutual connections based on expert judgments [19, 21]. The ADVIAN ${ }^{\circledR}$ has the capability of analyzing all interrelationships in diverse cross-impact matrices along with being able to perform supplementary measures such as criticality, stability, integration, driven, driving and precarious $[5,18,19]$.

\section{RESUlTS OF ANALYSIS}

The cross-impact matrix data for this study were collected from nine financial system experts using an online survey instrument to elicit their judgements of 20 CSFs described in Table I. These 20 factors were obtained from 205 factors reported in 127 related research papers on ERP system implementation and categorized into four categories of resource. (data, valuable, infrastructure, consultant and support in terms of management support, vendor support and training support), culture (communication, commitment, change, participation and values), project (implementation, team, leader and goals) and process (customization, package, plan and evaluation). Frequency distribution based on the number of previous research papers that cited a factor as being critical was constructed and normalized to realize a probability distribution. A mean probability threshold value of 0.88 was calculated to select 20 factors above the threshold value (Epizitone and Olugbara, 2019). Thereafter, these factors were presented to the experts to generate impact scores of factors. An understanding was reached among all participating experts by providing them with the necessary information and framework of the study. Given the nature of the study coupled with timeframe constraint, a minimum of 4 or 5 participants was accepted in the previous studies for impact score evaluation $[8,22,23]$. In this study, nine experts were engaged to provide their opinions on interrelationships of CSFs for implementing an ERP system in relation to financial functions. The number of participating experts is deemed acceptable for the research work of this nature.

During the process of data collection, an individual expert can use the online survey instrument administered to them to create several lists of preference chains of factors as suggested by Thompson, Olugbara and Singh [5]. The preference list approach enables the experts to list factors that are judged to be associated by transitive preference relation. The online survey instrument will then automatically process the numerous preference lists of factors created by experts to hatch a normalized cross-impact matrix (CIM) as shown in Table II.

\section{A. Factor Relationships}

The application of ADVIAN@ method has yielded results that have provided useful insights into the interrelationships of CSFs. The normalized CIM has made it possible to assess interrelationships that revealed a maximum direct active sum of 14.11 and maximum direct passive sum of 7.78 to dictate how the investigated factors impact the implementation of financial ERP system at varying levels. Table III presents further values of the average by two third of the standard deviation to comprehensively assess the characteristics of CSFs for a more detailed analysis. Emphasis was placed on CSFs whose values exceed the average by two third of the standard deviation to highlight significance of factors for implementing ERP systems [5, 19]. To supplement the assessment of CSFs, an additional analysis has been performed to consider factors that felt below the average not as much as two-third of the standard deviation. The interrelationship between two factors is usually expressed by active sum and passive sum that provide insightful knowledge about impacts of CSFs.

Direct relationship as demonstrated by active sum reveals the degree to which a CSF impacts on the implementation of financial ERP systems. Whereas passive sum demonstrates indirect relationship as the degree to which a financial ERP system impacts on CSFs. Relative scores were obtained by converting active sum and passive sum to percentile scale of 100 from 0 to permit the use of any number of factors in a system. The computational results based on the metrics of ADVIAN® are shown in Table III, where ellipses of DAS, RDAS, DPS, RDPS, RIAS, RIPS, stand for direct active sum, relative direct active sum, direct passive sum, relative direct passive sum, relative indirect active sum, and relative indirect passive sum respectively. Direct relationships produce high relative scores of 100 for active sum in the case of factor F1. It has a maximum impact on the financial system based on its impact on other factors in the system. Factor F17 gave a score of 48.82 relative direct active sum while factors F3 and F4 followed with the same value of 46.45 . For the passive sum that divulges the factors that are directly influenced and affected by other factors in the system, F14 has the highest relative direct passive sum of 100 to indicate that it is the most affected and influenced by other factors. F12 has a relative direct passive sum of 88.57 while three factors F6, F11 and F13 have relative direct passive sum of $84.28,82.85$ and 81.43 respectively.

The average relative scores for the first order are 36.06 and 65.42 for relative active sum and relative passive sum. The factors that exceeded the average by two-third of the standard deviation for either passive sum or active sum have an adverse effect on the other factors. F1 with maximum relative active sum has the lowest relative passive sum. The relative active sums for the factors F11, F12, F14 and F18 fall below the average and less the two third of the standard deviation (23.33). However, they have high values that exceeded the 79.31 average by two-third of the standard deviation for relative passive sum to reveal the degree of relationships that exist in the ERP system. This result indicates that a factor with high degree of direct impact on system has less influence on other factors. F1 has the strongest impact exerted on other factors but is less influenced by other factors with a value of 100 to 5.86. The impact of F1 can be clearly identified as shown in Table III to be less significant but has the strongest impact on the system. The factors F4, F8, F9 and F13 are highly influenced by F1. The most significant impact on factor F14 emanated from factors F1, F3, F4, F5, F7, F10 and F19. 
TABLE I. CATEGORY AND DESCRIPTION OF FACTORS

\begin{tabular}{|c|c|c|}
\hline Factor & Category & Description \\
\hline $\mathrm{F} 1$ & Resource & Top management support and commitment \\
\hline $\mathrm{F} 2$ & Culture & Interdepartmental communication and cooperation throughout the institution \\
\hline F3 & Culture & Commitment to business process reengineering to do away with redundant processes \\
\hline $\mathrm{F} 4$ & Project & Implementation of project management from initiation to closing \\
\hline F5 & Culture & Change management program to ensure awareness for any changes that may happen \\
\hline F6 & Project & Project team competence, formulation, composition, and involvement \\
\hline F7 & Resource & Education and training for stakeholders, including end users, technical and IT staff \\
\hline F8 & Project & Project champion presence to lead the implementation, authorized to use internal and external resources to complete implementation \\
\hline F9 & Project & Project mission and goals for the system with clear objective agreed upon \\
\hline F10 & Resource & ERP expert consultant use to guide the implementation process \\
\hline F11 & Process & Minimum level of customization to utilize ERP functionalities to a maximum \\
\hline F12 & Process & Package selection, carefully and professionally selected \\
\hline F13 & Culture & Understanding the institutional culture, norms, values, and beliefs \\
\hline F14 & Culture & User involvement and participation throughout implementation \\
\hline F15 & Resource & ERP vendor support and partnership \\
\hline F16 & Process & Business vision and plan \\
\hline F17 & Resource & Adequate IT infrastructure \\
\hline F18 & Process & Monitoring management especially evaluation of performance metrics \\
\hline F19 & Resource & Allocating and dedicating valuable resources \\
\hline F20 & Resource & Data management plan that ensures that data are accurately and efficient migrated to a new system and analysed properly \\
\hline
\end{tabular}

TABLE II. NORMALIZED CROSS-IMPACt MATRIX OF 20 FACTORS

\begin{tabular}{|c|c|c|c|c|c|c|c|c|c|c|c|c|c|c|c|c|c|c|c|c|}
\hline Factor & $\mathrm{F} 1$ & F2 & F3 & $\mathrm{F} 4$ & F5 & F6 & F7 & F8 & F9 & F10 & F11 & $\mathrm{F} 12$ & F13 & F14 & F15 & F16 & F17 & F18 & F19 & F20 \\
\hline $\mathrm{F} 1$ & 0.0 & 1.0 & 1.0 & 0.7 & 0.8 & 0.9 & 0.9 & 0.7 & 0.7 & 0.9 & 0.4 & 0.6 & 0.9 & 0.7 & 0.8 & 0.7 & 1.0 & 0.3 & 0.7 & 0.7 \\
\hline F2 & 0.0 & 0.0 & 0.4 & 0.2 & 0.2 & 0.4 & 0.3 & 0.4 & 0.2 & 0.4 & 0.3 & 0.3 & 0.4 & 0.3 & 0.3 & 0.2 & 0.2 & 0.2 & 0.1 & 0.2 \\
\hline F3 & 0.0 & 0.3 & 0.0 & 0.2 & 0.4 & 0.3 & 0.6 & 0.3 & 0.3 & 0.3 & 0.3 & 0.6 & 0.4 & 0.6 & 0.3 & 0.3 & 0.3 & 0.2 & 0.2 & 0.3 \\
\hline F4 & 0.1 & 0.4 & 0.3 & 0.0 & 0.4 & 0.6 & 0.3 & 0.4 & 0.1 & 0.3 & 0.4 & 0.3 & 0.2 & 0.6 & 0.4 & 0.2 & 0.2 & 0.3 & 0.2 & 0.4 \\
\hline F5 & 0.0 & 0.3 & 0.4 & 0.1 & 0.0 & 0.3 & 0.3 & 0.2 & 0.2 & 0.4 & 0.3 & 0.4 & 0.1 & 0.7 & 0.2 & 0.3 & 0.2 & 0.2 & 0.1 & 0.4 \\
\hline F6 & 0.0 & 0.1 & 0.2 & 0.0 & 0.2 & 0.0 & 0.0 & 0.6 & 0.2 & 0.2 & 0.3 & 0.2 & 0.2 & 0.3 & 0.1 & 0.1 & 0.0 & 0.2 & 0.0 & 0.2 \\
\hline F7 & 0.0 & 0.4 & 0.3 & 0.1 & 0.3 & 0.6 & 0.0 & 0.2 & 0.2 & 0.4 & 0.6 & 0.4 & 0.2 & 0.6 & 0.3 & 0.3 & 0.3 & 0.3 & 0.2 & 0.3 \\
\hline F8 & 0.1 & 0.1 & 0.2 & 0.1 & 0.2 & 0.1 & 0.1 & 0.0 & 0.4 & 0.3 & 0.2 & 0.3 & 0.3 & 0.3 & 0.2 & 0.1 & 0.1 & 0.2 & 0.2 & 0.2 \\
\hline F9 & 0.1 & 0.2 & 0.2 & 0.3 & 0.2 & 0.3 & 0.2 & 0.2 & 0.0 & 0.4 & 0.2 & 0.4 & 0.3 & 0.3 & 0.2 & 0.1 & 0.3 & 0.3 & 0.3 & 0.2 \\
\hline F10 & 0.0 & 0.2 & 0.3 & 0.1 & 0.1 & 0.2 & 0.1 & 0.1 & 0.1 & 0.0 & 0.3 & 0.6 & 0.4 & 0.6 & 0.3 & 0.3 & 0.3 & 0.2 & 0.3 & 0.2 \\
\hline F11 & 0.0 & 0.1 & 0.1 & 0.0 & 0.0 & 0.1 & 0.1 & 0.1 & 0.1 & 0.0 & 0.0 & 0.3 & 0.1 & 0.2 & 0.0 & 0.0 & 0.0 & 0.2 & 0.0 & 0.1 \\
\hline $\mathrm{F} 12$ & 0.0 & 0.1 & 0.1 & 0.0 & 0.0 & 0.1 & 0.1 & 0.1 & 0.1 & 0.0 & 0.2 & 0.0 & 0.1 & 0.1 & 0.1 & 0.1 & 0.2 & 0.2 & 0.2 & 0.2 \\
\hline F13 & 0.1 & 0.2 & 0.2 & 0.1 & 0.3 & 0.3 & 0.3 & 0.2 & 0.2 & 0.3 & 0.2 & 0.3 & 0.0 & 0.3 & 0.2 & 0.1 & 0.1 & 0.3 & 0.4 & 0.3 \\
\hline F14 & 0.0 & 0.2 & 0.0 & 0.0 & 0.0 & 0.2 & 0.0 & 0.2 & 0.1 & 0.0 & 0.2 & 0.2 & 0.3 & 0.0 & 0.1 & 0.1 & 0.0 & 0.2 & 0.0 & 0.3 \\
\hline F15 & 0.0 & 0.3 & 0.3 & 0.0 & 0.3 & 0.4 & 0.1 & 0.3 & 0.2 & 0.3 & 0.4 & 0.4 & 0.6 & 0.4 & 0.0 & 0.4 & 0.1 & 0.3 & 0.4 & 0.3 \\
\hline F16 & 0.0 & 0.3 & 0.3 & 0.1 & 0.2 & 0.3 & 0.2 & 0.3 & 0.3 & 0.2 & 0.4 & 0.4 & 0.6 & 0.3 & 0.3 & 0.0 & 0.2 & 0.3 & 0.4 & 0.3 \\
\hline F17 & 0.0 & 0.4 & 0.3 & 0.1 & 0.3 & 0.6 & 0.4 & 0.3 & 0.2 & 0.3 & 0.4 & 0.3 & 0.6 & 0.3 & 0.6 & 0.4 & 0.0 & 0.4 & 0.4 & 0.2 \\
\hline F18 & 0.0 & 0.1 & 0.1 & 0.0 & 0.1 & 0.1 & 0.0 & 0.1 & 0.0 & 0.1 & 0.1 & 0.1 & 0.0 & 0.1 & 0.0 & 0.0 & 0.0 & 0.0 & 0.0 & 0.0 \\
\hline F19 & 0.0 & 0.3 & 0.2 & 0.1 & 0.2 & 0.3 & 0.1 & 0.2 & 0.1 & 0.3 & 0.3 & 0.2 & 0.3 & 0.6 & 0.2 & 0.1 & 0.1 & 0.3 & 0.0 & 0.3 \\
\hline F20 & 0.0 & 0.4 & 0.2 & 0.1 & 0.1 & 0.2 & 0.3 & 0.2 & 0.1 & 0.2 & 0.4 & 0.2 & 0.1 & 0.4 & 0.1 & 0.0 & 0.2 & 0.3 & 0.1 & 0.0 \\
\hline
\end{tabular}


TABLE III. DIRECT AND INDIRECT RELATIONSHIPS

\begin{tabular}{|c|c|c|c|c|c|c|c|c|c|c|}
\hline Factor & DAS & RDAS & $\begin{array}{c}\text { Ranking by } \\
\text { RDAS }\end{array}$ & DPS & RDPS & $\begin{array}{c}\text { Ranking by } \\
\text { RDPS }\end{array}$ & RIAS & $\begin{array}{l}\text { Ranking by } \\
\text { RIAS }\end{array}$ & RIPS & $\begin{array}{l}\text { Ranking by } \\
\text { RIPS }\end{array}$ \\
\hline F1 & 14.11 & 100.00 & 1 & 0.44 & 5.71 & 20 & 100.00 & 1 & 5.86 & 20 \\
\hline F2 & 5.56 & 39.37 & 9 & 5.89 & 75.71 & 6 & 36.01 & 7 & 69.06 & 9 \\
\hline F3 & 6.56 & 46.45 & 3 & 5.56 & 71.42 & 8 & 41.81 & 4 & 64.65 & 11 \\
\hline F4 & 6.56 & 46.45 & 4 & 2.44 & 31.43 & 19 & 41.86 & 3 & 25.91 & 19 \\
\hline F5 & 5.56 & 39.37 & 8 & 4.67 & 60.00 & 14 & 34.15 & 10 & 53.47 & 15 \\
\hline F6 & 3.33 & 23.62 & 16 & 6.56 & 84.28 & 3 & 19.16 & 16 & 78.44 & 5 \\
\hline F7 & 6.33 & 44.88 & 5 & 4.67 & 60.00 & 13 & 37.89 & 5 & 53.64 & 14 \\
\hline F8 & 4.11 & 29.13 & 14 & 5.44 & 70.00 & 10 & 27.67 & 13 & 69.87 & 8 \\
\hline F9 & 5.22 & 37.00 & 10 & 4.11 & 52.85 & 16 & 34.78 & 9 & 52.30 & 16 \\
\hline F10 & 5.00 & 35.43 & 11 & 5.78 & 74.28 & 7 & 30.48 & 12 & 67.77 & 10 \\
\hline F11 & 1.67 & 11.81 & 19 & 6.44 & 82.85 & 4 & 8.54 & 19 & 85.56 & 3 \\
\hline F12 & 2.22 & 15.75 & 18 & 6.89 & 88.57 & 2 & 13.67 & 17 & 92.00 & 2 \\
\hline F13 & 4.89 & 34.64 & 12 & 6.33 & 81.43 & 5 & 31.73 & 11 & 79.04 & 4 \\
\hline F14 & 2.33 & 16.53 & 17 & 7.78 & 100.00 & 1 & 12.76 & 18 & 100.00 & 1 \\
\hline F15 & 6.00 & 42.52 & 6 & 5.00 & 64.28 & 12 & 35.52 & 8 & 57.91 & 12 \\
\hline F16 & 5.89 & 41.73 & 7 & 4.11 & 52.85 & 17 & 36.12 & 6 & 48.61 & 17 \\
\hline F17 & 6.89 & 48.82 & 2 & 4.11 & 52.85 & 18 & 43.28 & 2 & 45.2 & 18 \\
\hline F18 & 1.00 & 7.09 & 20 & 5.44 & 69.99 & 11 & 5.79 & 20 & 74.65 & 6 \\
\hline F19 & 4.56 & 32.28 & 13 & 4.56 & 58.57 & 15 & 26.58 & 14 & 54.86 & 13 \\
\hline F20 & 4.00 & 28.34 & 15 & 5.56 & 71.42 & 9 & 23.68 & 15 & 71.36 & 7 \\
\hline Average & 5.09 & 36.06 & & 5.09 & 65.42 & & 32.07 & & 62.51 & \\
\hline STD DEV & 2.74 & 19.39 & & 1.62 & 20.83 & & 19.55 & & 21.85 & \\
\hline AVG+2/3std Dev & 6.91 & 48.99 & & 6.17 & 79.31 & & 45.11 & & 77.08 & \\
\hline AVG-2/3std Dev & 3.26 & 23.13 & & 4.01 & 51.54 & & 19.04 & & 47.94 & \\
\hline
\end{tabular}

\section{B. Classification of Factors}

The classification of CSFs can be done using the measures of criticality, integration, and stability for conditional state of system of factors. The state of a system can be significantly altered because of changes in any factors deem critical in the system. Table IV shows the computed values for criticality, integration and stability of factors based on the calculations of ADVIAN®.

There are significant changes in the system when there are changes to any of the CSFs based on their criticality. A high level of criticality was obtained for factors F2, F3 and F13. These factors have a high level of criticality, when looking at the average by the standard deviation. F3 has the highest criticality score of 51.99, followed by F13 with 50.08 and 49.87 for F2. These factors necessitate an early update for corrective measures to be taken should they change. A low level of criticality which is below 34.24 was obtained for factors F1, F4, F11 and F18, hence changes to these factors render minimal impacts on the system.

Fig. 1 shows the contour lines of the criticality corresponding to the system stability. However, the active sum and passive sum present the reverse dependency. This implies that factors with high criticality will have a low stability as in the case of F2, F3 and F

The connection of factors in the system can be determined by the level of integration. A high-level integration (55.39) for a factor indicates a strong connection with the rest of the system. A high value of integration was obtained for factor F13 while factor F14 gave a value of 56.38. The other factors with high integration are F1 (52.93), F2 (52.53), F3 (53.23) and F12 (52.83) with values above the 51.09 threshold. This presents the existence of a mutual connection and likely feedback loops among these factors. These feedback loops strongly reinforce each other mutually in indirect connections at different levels to confirm the presence of mutual connections and feedback loops among the factors as can be seen by the contour line of Fig. 2. In the integration system grid, high integration factors of F14, F13 and F1 have high passive sum, high active sum and low integration score with low passive sum and active sum.

The determination of system stability was based on the distribution of factors toward active sum and passive sum axes $[5,17-20]$. Factors that are controlled by the system are aligned close to the axis of the passive sum with low active sum, while factors that control the systems are closed to the 
active sum axis with low passive sum (Linss and Fried, 2010). Stability level attenuates feedback loops to ensure the absence of uncontrolled feedback loops $[5,17,19]$. A high stability of 73.08 of the average by two-third of the standard deviation was obtained. Factors F1, F11, F12, F14 and F18 contribute heavily to the system stability. F18 (89.26), F1 (88.92) and F $11(84.47)$ are factors with high stability values, followed by F12 (76.20) and F14 (77.36). The combinations of these factors with high passive sum and low active sum coupled with their different integration levels and high stability indicate that they are independent factors within the system which can hardly alter these factors. Fig. 3 shows the contour line for the system stability which position factors F4 and F6 above the system stability of 64.97 .

\section{Ranking of Factors}

Precarious, driving and driven are essential measures for ranking of CSFs. The precarious measure is obtained for a factor by calculating the geometric mean of the active indirect sum and criticality measurement. The driving measure of a factor considers the geometric mean of indirect sum without a complete percentage of the criticality measure. The driven measure for each factor is an inverse of the driving measure which substitutes the indirect active sum with passive sum. Table $\mathrm{V}$ shows the scores computed for precarious, driving and driven based on the ADVIAN@ method. Precarious CSFs exert utmost influence on the system and are affected by peripheral forces. The factors F1 (49.21), F2 (42.37), F3
(46.63) and F17 (43.75) present high precarious scores above the average by two third of the standard deviation of 41.40 . These factors have strong influence on the system and are invulnerable to external forces because they are not ideal to warrant intervening activities.

Fig. 4 presents the contour line for the determination of the most precarious factors and lowest precarious factors of F18, F11 and F14. High driving ranking for CSFs is essential for implementation success because these factors present high influence on other factors in the system and do not cause strong feedback. Factors of F1 (87.05), F4 (52.98) and F17 (49.13) are drivers with outstanding characteristics to drive a successful financial ERP system implementation. These factors are non-critical with high active sum, but they are good beginning point for intervention activities because of their suitability for external actions that dependent on the ability to influence other factors without causing strong feedback.

Fig. 5 shows the contours for driving factors. Driven impact factors are non-critical with high passive sums. They are more reactive in nature and are not reasonably altered by intervening activities. Nonetheless, they indicate the success of external actions taken on driving factors and not reasonably affected by external changes made to the system. Factors of F6, F11, F12, F14 and F18 are good indicators of success of external intervention on financial ERP system implementation by driving factors. Fig. 6 presents the contour lines for the driven ranking impact factors.

TABLE IV. ClasSIFICATION OF FACTORS By MEASURES OF CRITICALITY, INTEGRATION, AND STABILITY

\begin{tabular}{|c|c|c|c|}
\hline Factor & Critical & Integration & Stability \\
\hline F1 & 24.22 & 52.93 & 88.92 \\
\hline $\mathrm{F} 2$ & 49.87 & 52.53 & 52.67 \\
\hline F3 & 51.99 & 53.23 & 49.22 \\
\hline $\mathrm{F} 4$ & 32.93 & 33.88 & 68 \\
\hline F5 & 42.73 & 43.81 & 58.32 \\
\hline F6 & 38.77 & 48.8 & 69.2 \\
\hline F7 & 45.08 & 45.76 & 55.59 \\
\hline F8 & 43.97 & 48.77 & 60.36 \\
\hline F9 & 42.65 & 43.54 & 58.23 \\
\hline F10 & 45.45 & 49.12 & 57.95 \\
\hline F11 & 27.03 & 47.05 & 84.47 \\
\hline F12 & 35.46 & 52.83 & 76.2 \\
\hline F13 & 50.08 & 55.39 & 54.71 \\
\hline F14 & 35.73 & 56.38 & 77.36 \\
\hline F15 & 45.35 & 46.71 & 55.97 \\
\hline F16 & 41.9 & 42.37 & 58.55 \\
\hline F17 & 44.23 & 44.24 & 55.78 \\
\hline F18 & 20.79 & 40.22 & 89.25 \\
\hline F19 & 38.19 & 40.72 & 64.19 \\
\hline F20 & 41.11 & 47.52 & 64.44 \\
\hline Average & 39.88 & 47.29 & 64.97 \\
\hline STD DEV & 8.46 & 5.7 & 12.16 \\
\hline AVG+2/3std Dev & 45.52 & 51.09 & 73.08 \\
\hline AVG-2/3std Dev & 34.24 & 43.49 & 56.86 \\
\hline
\end{tabular}




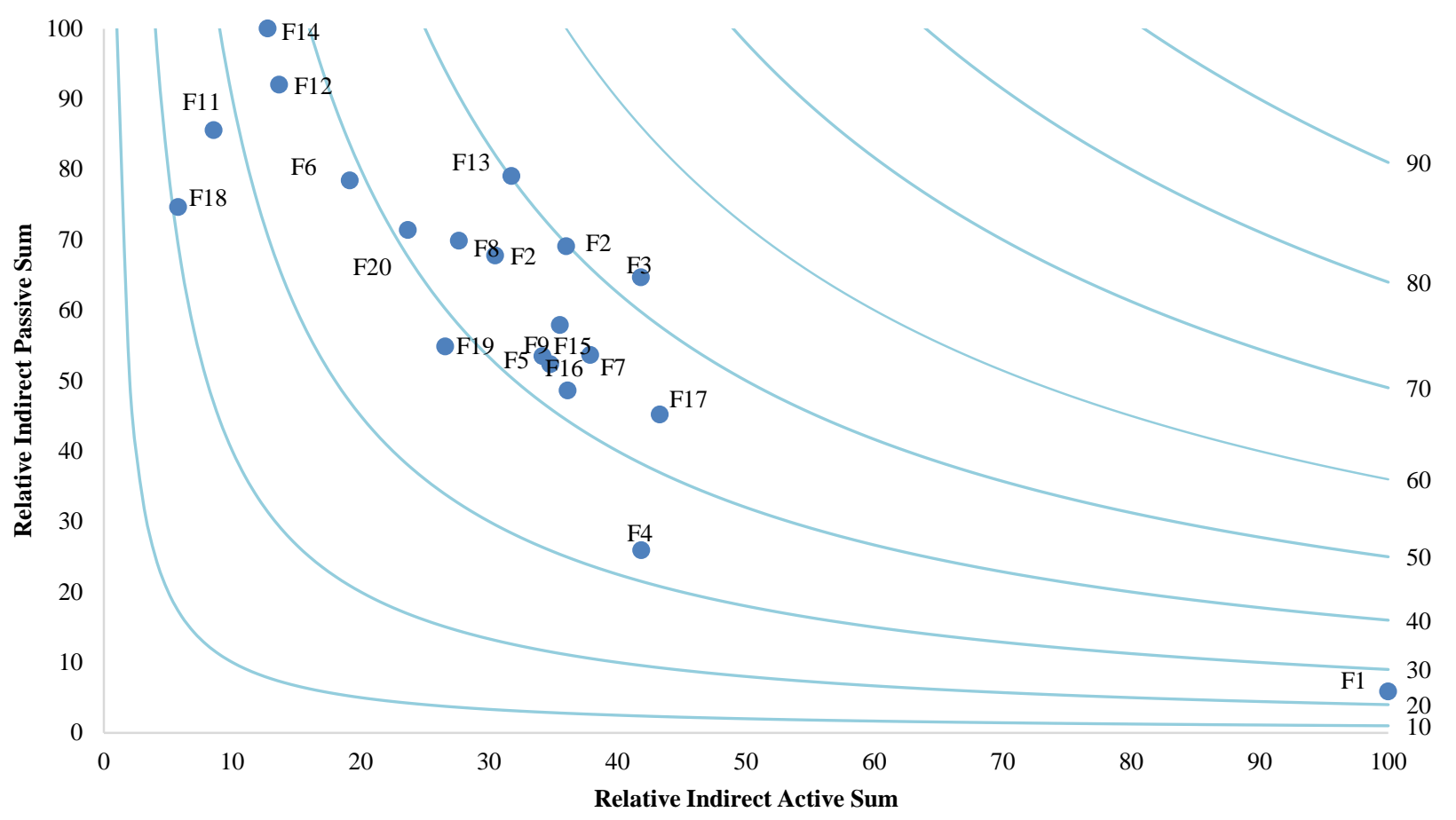

Fig. 1. Classification of Factors by a Measure of Criticality.

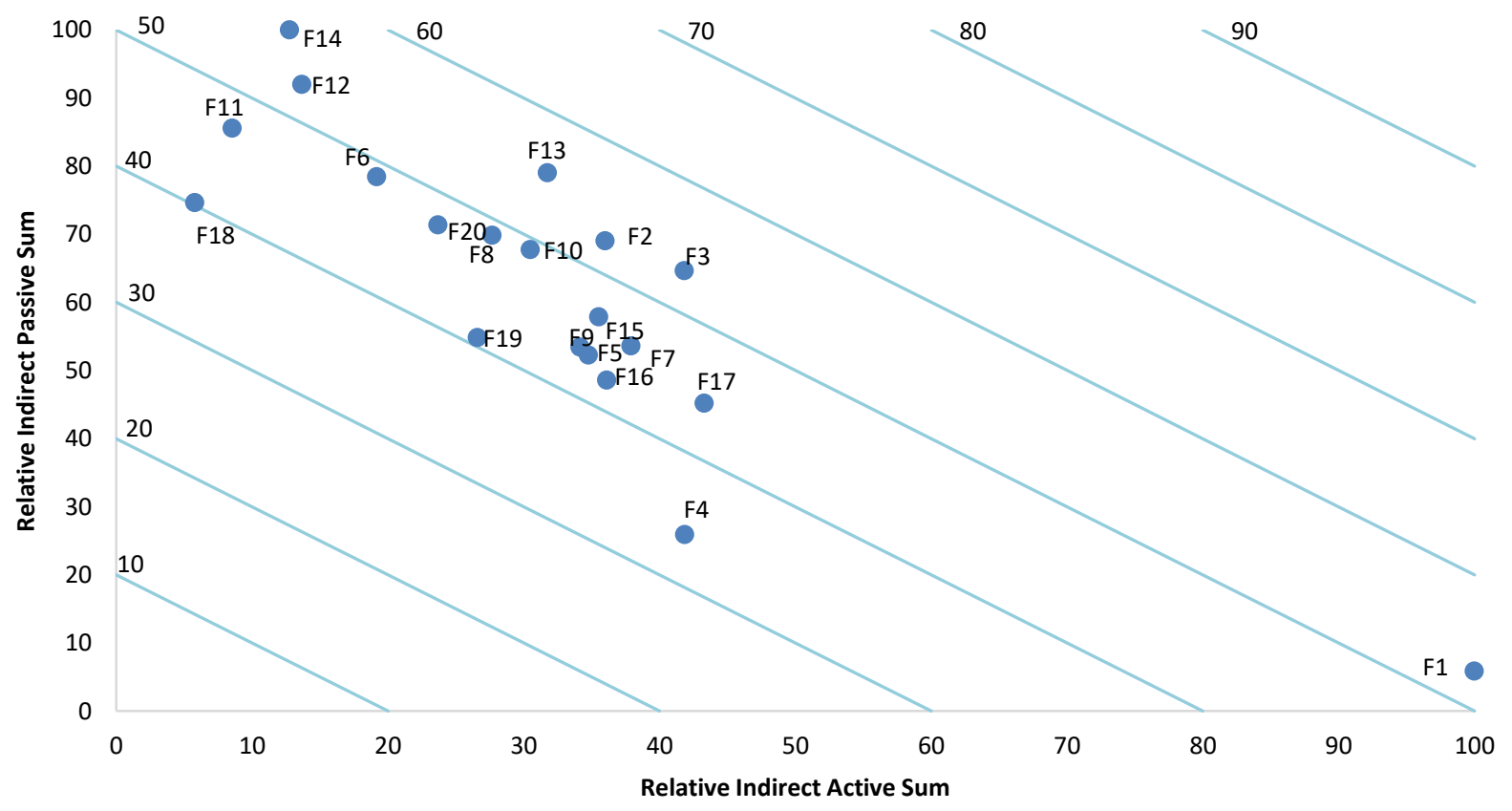

Fig. 2. Classification of Factors by a Measure of Integration. 


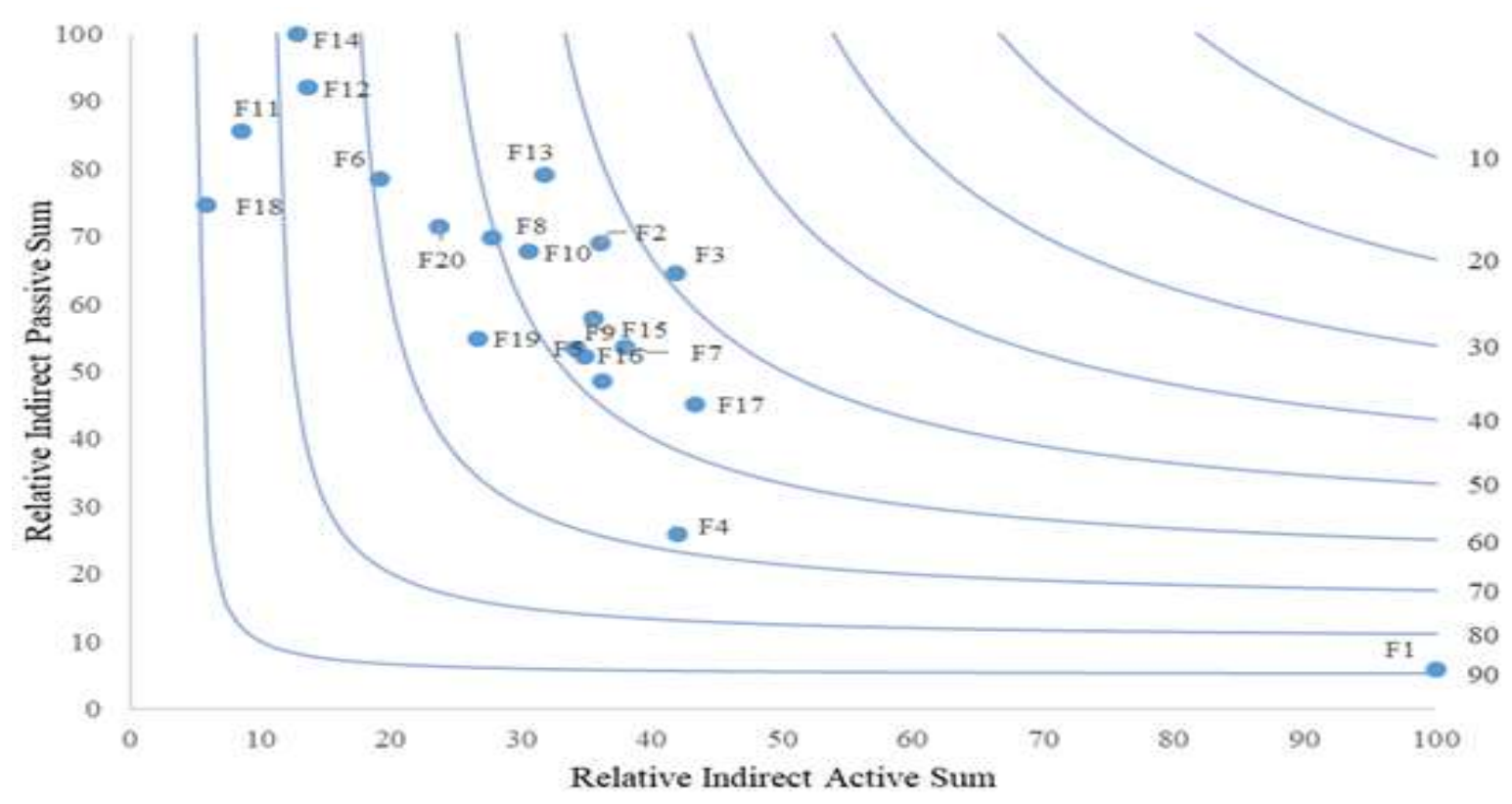

Fig. 3. Classification of Factors by a Measure of Stability.

TABLE V. RANKING OF FACtors by MEASURES of PRECARIOUS, DRIVING, AND DRIVEN

\begin{tabular}{|c|c|c|c|c|c|c|}
\hline Factor & Precarious & Precarious Ranking & Driving & Driving Ranking & Driven & Driven Ranking \\
\hline $\mathrm{F} 1$ & 49.21 & 1 & 87.05 & 1 & 21.08 & 20 \\
\hline $\mathrm{F} 2$ & 42.37 & 4 & 42.49 & 10 & 58.84 & 10 \\
\hline $\mathrm{F} 3$ & 46.63 & 2 & 44.80 & 6 & 55.71 & 13 \\
\hline $\mathrm{F} 4$ & 37.13 & 12 & 52.98 & 2 & 41.68 & 19 \\
\hline F5 & 38.20 & 10 & 44.22 & 8 & 55.34 & 14 \\
\hline F6 & 27.26 & 16 & 34.25 & 16 & 69.30 & 5 \\
\hline F7 & 41.33 & 5 & 45.62 & 5 & 54.27 & 16 \\
\hline F8 & 34.88 & 13 & 39.37 & 14 & 62.57 & 8 \\
\hline F9 & 38.51 & 9 & 44.66 & 7 & 54.77 & 15 \\
\hline F10 & 37.22 & 11 & 40.78 & 11 & 60.80 & 9 \\
\hline F11 & 15.20 & 19 & 24.97 & 19 & 79.01 & 2 \\
\hline F12 & 22.01 & 17 & 29.70 & 17 & 77.06 & 3 \\
\hline F13 & 39.87 & 7 & 39.80 & 13 & 62.81 & 7 \\
\hline F14 & 21.35 & 18 & 28.64 & 18 & 80.17 & 1 \\
\hline F15 & 40.14 & 6 & 44.06 & 9 & 56.26 & 12 \\
\hline F16 & 38.90 & 8 & 45.81 & 4 & 53.14 & 17 \\
\hline F17 & 43.75 & 3 & 49.13 & 3 & 50.21 & 18 \\
\hline $\mathrm{F} 18$ & 10.97 & 20 & 21.42 & 20 & 76.89 & 4 \\
\hline F19 & 31.86 & 14 & 40.54 & 12 & 58.23 & 11 \\
\hline F20 & 31.20 & 15 & 37.34 & 15 & 64.83 & 6 \\
\hline Average & 34.40 & & 41.88 & & 59.65 & \\
\hline STD DEV & 10.28 & & 13.36 & & 13.72 & \\
\hline AVG+2/3std Dev & 41.25 & & 50.79 & & 68.80 & \\
\hline AVG-2/3std Dev & 27.55 & & 32.97 & & 50.50 & \\
\hline
\end{tabular}




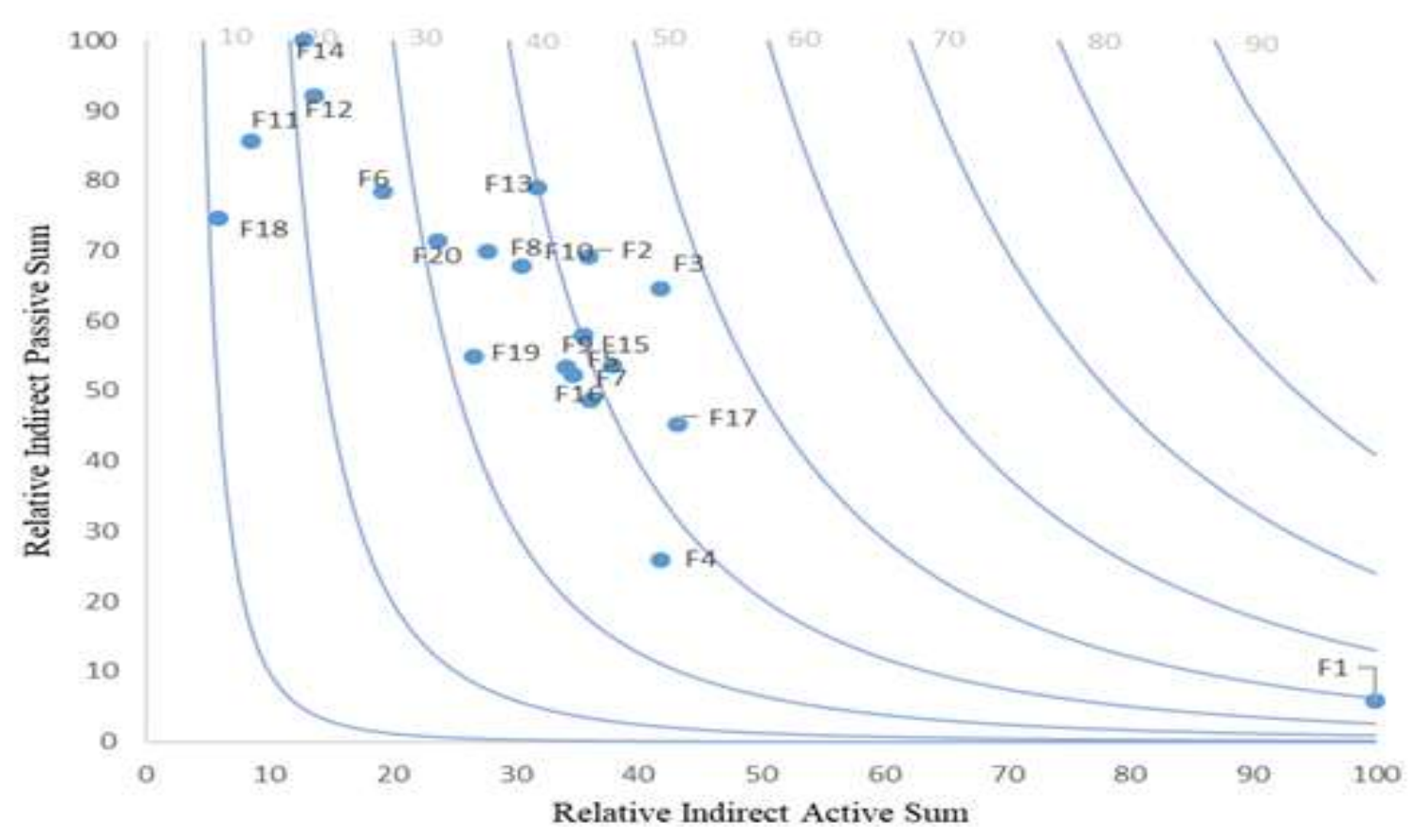

Fig. 4. Ranking of Factors by a Measure of Precarious.

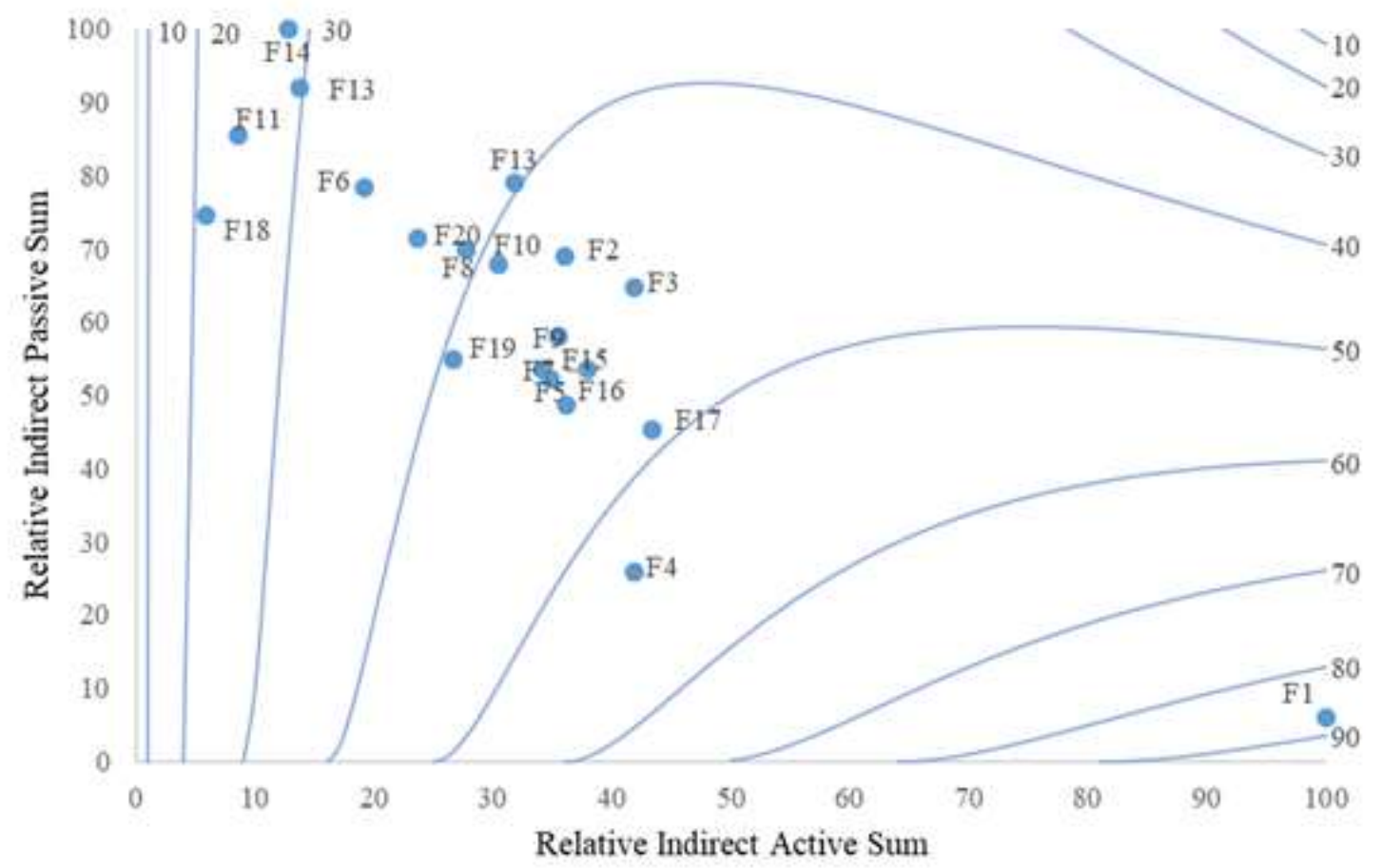

Fig. 5. Ranking of Factors by a Measure of Driving. 


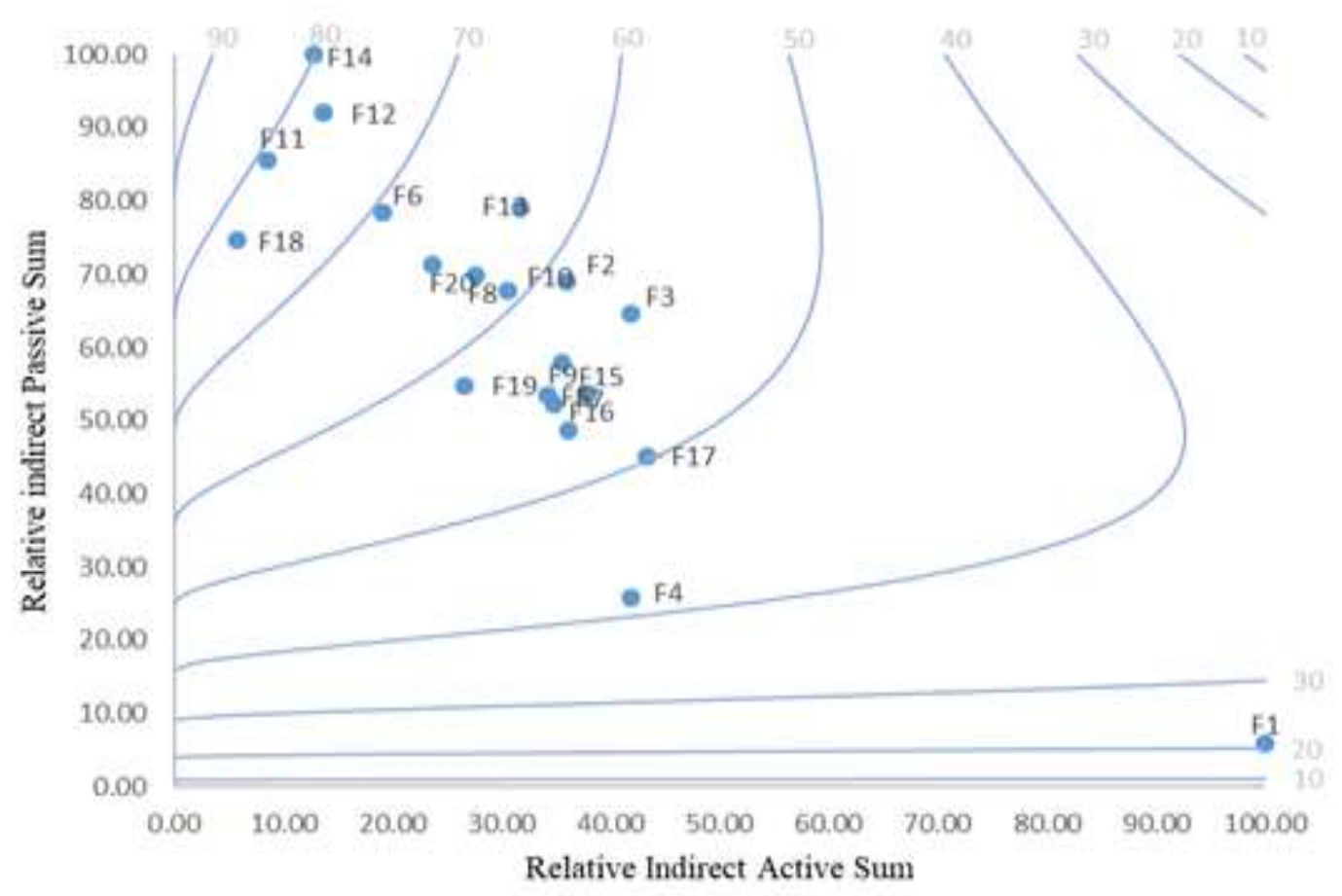

Fig. 6. Ranking of Factors by a Measure of Driven.

\section{DISCUSSION OF RESULTS}

The objective of this study was to use the ADVIAN® method to determine factors that are critical for implementing ERP systems to support financial functions. Consequently, 20 top CSFs aggregated from the literature were subjected to ADVIAN®. The exploration of these factors has yielded valuable insights to the successful ERP system implementation that would support financial functions in a HEI. In this study, top management support and commitment (F1) is highly relevant for implementing financial ERP systems. Given that this factor has been cited by several authors in the general case of ERP system implementation is not a coincidence. The factor means authorizing, commissioning, and making available resources that are needed for the implementation of a financial ERP system. A successfully implemented financial ERP system is an asset to top management who rely heavily on outputs of the system to support strategic decision making in an organization. Despite this result, literature has reported the dysfunctionality of ERP system in financial reporting, which requires that attention be given to this factor [24]. The factor presents a high active sum of 100, stability of 88.92 and driving score of 87.05. Despite the low criticality of this factor, any changes presented to it would need intervening activities because it has great impact on other factors in the system. Hence, mirroring the previous and current studies for successful implementation of ERP systems [25-27].

Interdepartmental communication and cooperation (F2), is highly critical for consideration in the implementation of a financial ERP system. The need for open communication and cooperation among different stakeholders cannot be overlooked. Failure to properly communicate and cooperate is likely to cause system failure $([10,28,29]$. This factor presents a high passive sum of 75.71 and active sum of 39.37 in explaining the high criticality of 49.87 and precarious ranking of 42.37. This result makes it of great influence because of its dependency on other factors and is strongly influenced by other factors of the system. A high driving value of 42.49 is a clear indication that is it critical. Commitment to business process reengineering to do away with redundant processes (F3) has been found to be critical to financial ERP system implementation where functions have been highly automated. Business process reengineering refers to the restructuring of business processes in an organization. The factor presents a high passive sum of 71.42, which indicates the impact that other factors exert on it. In addition, the factor reflected a high criticality score of 51.99 and integration of 53.23. These values indicate the contributions of the factor to successful implementation of ERP system to support financial functions. This study converges with previous studies that cited this factor as critical, given its classification as an important integrator in the system with high precarious score of 46.63 is an indication of strong influence on the system. Moreover, a driven score of 44.80 above the average makes it critical as one prime organizational resource for improvement that warrants an intervention activity should there be any change to it $[5,30]$.

The implementation of project management from the initiation phase to the closing (F4) was identified in this study to be a factor with high stability score of 68.00 , which implies its significant contribution to the stability of an ERP system implementation. The driving value of 52.98 was obtained for the factor that alludes to its criticality in a previous study and highlights its significance in the success of financial ERP system because of being active ([1]. Project management entails a broader scope of implementing ERP systems to support financial functions. For the financial system to be 
successful, a good project management plan must be instituted. To promote successful financial system implementation, it is important to build team, deal with conflict and execute objectives of the implementation [31]. It is important to focus attention on the implementation of a financial system given that ERP project can tend to be complex $[32,33]$.

Change management program to build awareness and ensure readiness for changes that may happen (F5) was identified in this study to be critical. Presenting high value of 39.37 for active sum and high value of 44.22 for driving than the average. It presents itself to be active in the system implementation and impacting other factors in the system. Change spans a great length ranging from cultural, organizational, and structural changes and therefore, a stable and successful setting is required obviously for successful implementation of ERP system [34-37]. Project team competence of formulation, composition and involvement (F6) has emerged in this study to concretely align with previous studies as a factor of great influence on the system because of other factors such as F1, F4, F7 and F17 that significant impact on it. It has low precarious value of 27.26 indicating that it can be used for intervention given that it may be suitably influenced by external actions. Project team plays an important role in the system implementation indicating that formation, participation, skills and involvement is highly relevant for the success of implementing an ERP system [3438]. Rightly deploying a project team with competency to execute specific tasks can mediate a successful financial ERP system, given that it has a high driven score of 69.30. Hence, it is a good indicator of successful intervention that requires attention of management.

Education and training for stakeholders including end users, technical and information technology staff (F7) is deemed to be critical in literature on ERP systems. Lack of education can be directly attributed to many implementation challenges such as system failure, employee uneasiness with using the system and training overhead that have been reported in literature $[30,34,35,39,40]$. The precarious value of 41.33 implies that this factor is a precarious impact factor with high activities that require actions to be taken during the implementation should there be any issue affecting it. Project champion to lead the financial system implementation, authorized to use internal and external resources to complete financial ERP system implementation (F8) is important. The significance of ERP project champion is recognized in terms of a precarious value of 34.88 that indicates its influence on the system. This study deemed this factor significant because of the driven nature with a value of 62.57 , which makes it a good indicator of success and integration score of 48.77 requires that it be monitored.

Project mission and goals for the system with clear objective agreed upon (F9) is rank 9th place to be precarious (38.51) and 7th to be driving (44.66). This supports the listing of the factor as critical to financial ERP system implementation in accordance with previous literature. The factor will require corrective actions and interventions if there is any deviation from the goals and mission of the project that will adversely affect implementation. Consequently, it alludes to the need for a clear future plan for implementing financial ERP systems as echoed in the previous studies [2, 4, 5]. ERP expert consultant use to guide the implementation process (F10) of financial ERP systems is critical with a value of 45.45 above the average and minimum threshold of 34.21. Driven value of 60.80 computed for this factor coupled with a high passive sum of 74.28 support the previous studies that have highlighted it as a success indicator. The presence of this factor in a feedback loop is highly probable as previous studies link its inclusion in implementation to avoid failure and transfer knowledge to the implementation team $[1,41]$.

Minimum level of customization to utilize ERP functionalities to a maximum $(\mathrm{F} 11)$ is required for a successful implementation of financial systems. A high direct passive sum of 82.85 and high indirect passive sum of 85.56 signify high influence of the factor on implementation of financial ERP systems. This is supported by high stability value of 84.47 to indicate that it contributes highly to system stability which is also an indication of the strong impact that other factors in the system have on it. A driven score of 79.01 indicates further that the factor is strongly impacted on by other factors in the financial system which is an indication of success of intervention activities on the system. In addition, a very low precarious score of 15.20 attests to its stability in the system. Previous studies have posited that minimal level of customization should be instituted to prevent delays and dissatisfaction of ERP systems which indirectly implies the need for a vanilla financial ERP system [1, 42-44].

Package selection carefully and professionally selected (F12) for financial ERP systems is a highly significant factor with a driven score of 77.06. This score makes it a success gage of measure of intervention because it has most influenced on the system and is guided by internal impacts on the system. It contributes significantly to the stability of a financial ERP system implementation with a value of 76.20. Previous studies have revealed the focus on selecting the right package to be pivotal to successful implementation of ERP systems [1, 32, 38, 41]. The integration score of 52.83 substantiates the previous studies to postulate the right package selection for financial ERP system to be critical for implementation success.

Understanding the institutional culture which include the norms, values, and beliefs (F13) is regarded as important in this study in terms of criticality (50.08) and integration (55.39). The high passive sum of 62.81 for this factor leads to it been ranked as driven factor which aligns with the preceding studies that have considered it to be highly critical for successful implementation of ERP systems [1, 4, 5, 8, 13, 45, 46]. Consequently, in the context of financial ERP systems, the factor requires a monitoring intervention to be deployed. User involvement and participation throughout the implementation $(\mathrm{F} 14)$ process of financial ERP systems has been established with a 100 percent passive sum, irrespective of low active sum. This factor presents high stability score of 77.36 and driven score of 80.17 which make it a major success factor for the implementation of financial ERP systems. The integration score of 56.38 supports the stability and driven measures of this factor with a low precarious impact score of 21.35. In this study, we have identified the factor to be highly 
influential and driven in nature for monitoring the implementation of financial ERP systems because of its dependency on other factors [5].

ERP vendor support and partnership (F15) has emerged for a financial system implementation with a criticality score of 45.35. However, the low precarious score indicates that it is relevant for intervention because of its high reactive and driving score of 44.06. Previous authors have supported this factor to be important for the successful implementation of ERP systems in form of technical assistance, system maintenance, system update and user training [5, 42]. Business vision and plan (F16) for implementation of a financial ERP system presents low ranking and classification scores below the average values. However, the factor is reactive with an active sum of 41.73 and indirect passive sum of 48.61 above the average less the two-third standard deviation. This indicates that it impacts other factors in the system, it should be monitored and can be used for interventions because of its driving nature (45.81). In addition, a criticality score of 41.90 is observed in the system. A plan with clear vision and agreed upon objectives is reported by previous authors to be important for the successful implementation of ERP systems [9, 23, 34, 37, 47-53].

Adequate IT infrastructure (F17) for implementing financial ERP systems has emerged to be critical with a high driving score of 49.13, criticality score of 44.23 and precarious score of 43.75 over the average. It is an indication that it is reactive in nature and strongly influencing the system which make it suitable for interventions. Prior studies have ranked the adequacy of IT infrastructure high across implementations to indicate its significance $[23,50]$. Monitoring management especially evaluation of performance metrics (F18) is established in this study as driven in nature with a score of 76.89. Its high passivity indicates dependency on other factors in the system and provides an enabler of high stability (89.25). The low precarious score of 10.97 implies it could be used for intervention. This study upholds the prior studies that have elevated this factor to be critical for preventing adverse consequences for the implementation of ERP systems [4, 23, 50].

Allocating and dedicating valuable resources (F19) for implementing financial ERP systems is presented in this study to contradict the results of previous studies that have judged it as critical $[54,55]$. Both low values for passive sum and active sum indicate that the role of this factor is neutral with no significant changes. Hence, its presence could likely contribute to the system stability (64.19). It is also an indication that this factor is torpid in nature. Data management plan that ensures that data are accurately and efficiently migrated to a new system and analyzed properly (F20) is presented in this study to be dependent on other factors in the system because of its high passive scores of 71.42 and 71.36. The integration score of 47.52 and critically score of 41.11 have established this factor to be important for implementing financial ERP systems. It is being driven in nature (64.83), it can indicate success, and offer stability (64.44) because of its low precarious score of 37.34 .

In this study, CSFs for implementing financial ERP systems have been ranked and classified to structure a comprehensive model (Table VI) that can help organization to mediate a successful system implementation that takes into account the identified factors for supporting financial functions.

TABLE VI. ADVIAN® ANALYSIS OF FACtors, Highly CRITICAL $(\sqrt{ })$, ABOVE AVERAGE $(\sqrt{ })$

\begin{tabular}{|c|c|c|c|c|c|c|c|c|}
\hline \multirow[b]{2}{*}{ Factor } & \multicolumn{2}{|l|}{ Interaction } & \multicolumn{3}{|c|}{ Classification } & \multicolumn{3}{|l|}{ Ranking } \\
\hline & Highly Active & $\begin{array}{l}\text { Highly } \\
\text { Passive }\end{array}$ & Criticality & Integration & Stability & Precarious & Driving & Driven \\
\hline F1 & $\sqrt{ } \sqrt{ }$ & & & $\sqrt{ } \sqrt{ }$ & $\sqrt{ } \sqrt{ }$ & $\sqrt{ } \sqrt{ }$ & $\sqrt{ } \sqrt{ }$ & \\
\hline $\mathrm{F} 2$ & $\sqrt{ }$ & $\sqrt{ }$ & $\sqrt{ } \sqrt{ }$ & $\sqrt{ } \sqrt{ }$ & & $\sqrt{ } \sqrt{ }$ & $\sqrt{ }$ & \\
\hline F3 & $\sqrt{ }$ & $\sqrt{ }$ & $\sqrt{ } \sqrt{ }$ & $\sqrt{ } \sqrt{ }$ & & $\sqrt{\sqrt{ }}$ & $\sqrt{ }$ & \\
\hline $\mathrm{F} 4$ & $\sqrt{ }$ & & & & $\sqrt{ }$ & $\sqrt{ }$ & $\sqrt{ } \sqrt{ }$ & \\
\hline F5 & $\sqrt{ }$ & & $\sqrt{ }$ & & & $\sqrt{ }$ & $\sqrt{ }$ & \\
\hline F6 & & $\sqrt{ } \sqrt{ }$ & & $\sqrt{ }$ & $\sqrt{ }$ & & & $\sqrt{ } \sqrt{ }$ \\
\hline F7 & $\sqrt{ }$ & & $\sqrt{ }$ & & & $\sqrt{ } \sqrt{ }$ & $\sqrt{ }$ & \\
\hline F8 & & $\sqrt{ }$ & $\sqrt{ }$ & $\sqrt{ }$ & & $\sqrt{ }$ & & $\sqrt{ }$ \\
\hline F9 & $\sqrt{ }$ & & $\sqrt{ }$ & & & $\sqrt{ }$ & $\sqrt{ }$ & \\
\hline F10 & & $\sqrt{ }$ & $\sqrt{ }$ & $\sqrt{ }$ & & & & $\sqrt{ }$ \\
\hline F11 & & $\sqrt{ } \sqrt{ }$ & & & $\sqrt{ } \sqrt{ }$ & & & $\sqrt{ } \sqrt{ }$ \\
\hline F12 & & $\sqrt{ } \sqrt{ }$ & & $\sqrt{ } \sqrt{ }$ & $\sqrt{ } \sqrt{ }$ & & & $\sqrt{\sqrt{ }}$ \\
\hline F13 & & $\sqrt{ } \sqrt{ }$ & $\sqrt{ } \sqrt{ }$ & $\sqrt{ } \sqrt{ }$ & & $\sqrt{ }$ & & $\sqrt{ }$ \\
\hline F14 & & $\sqrt{ } \sqrt{ }$ & & $\sqrt{ } \sqrt{ }$ & $\sqrt{ } \sqrt{ }$ & & & $\sqrt{ } \sqrt{ }$ \\
\hline F15 & $\sqrt{ }$ & & $\sqrt{ }$ & & & $\sqrt{ }$ & $\sqrt{ }$ & \\
\hline F16 & $\sqrt{ }$ & & $\sqrt{ }$ & & & $\sqrt{ }$ & $\sqrt{ }$ & \\
\hline F17 & $\sqrt{ }$ & & $\sqrt{ }$ & & & $\sqrt{\sqrt{ }}$ & $\sqrt{ }$ & $\sqrt{ } \sqrt{ }$ \\
\hline F18 & & $\sqrt{ }$ & & & $\sqrt{ } \sqrt{ }$ & & & \\
\hline F19 & & & & & & & & \\
\hline F20 & & $\sqrt{ }$ & $\sqrt{ }$ & $\sqrt{ }$ & & & & $\sqrt{ }$ \\
\hline
\end{tabular}


The ranking has provided suitable factors that should be selected for system improvement and control of improvement success. The model has considered factors with the highest driving scores to be the best impact factors for intervention changes. Furthermore, selecting a smaller number of low precarious factors along with high driven impact factors as success indicators of intervention activities. The classification of CSFs for implementing financial ERP systems has been achieved and determined by their integration into the system as well as contribution to the system stability while proving the identified critical factors. The classification of factors has served as a good basis for observation with care of implementing financial ERP systems. The most significant measures of successful implementation of ERP systems are driven and driving CSFs. The driving CSFs exert impacts on the system which implies they should be considered as a good starting point for intervening activities. Hence, they are prime resources in an organization for improvement. However, a small number of the selected CSFs can be made based on the exclusion of high precarious CSFs when there are too high driving factors for suitable improvement. Furthermore, driven CSFs are the most influential in the system which makes them good indicators of success for intervening activities taken on driving resources. These factor characteristics make conditions of driven resources a good measurement of success for intervention activities and state of driving resources can be improved by intervention activities.

\section{CONCLUSION}

This study identifies the CSFs for successful implementation of an ERP system to support financial functions. This study has investigated the CSFs of ERP system implementation to support financial functions in the context of HEI using the ADVIAN® method. Our study delivers stimulating insights into the mindset regarding CSFs and implementation of financial ERP systems in the HEIs. This study has employed a rigorous scientific method to generally contribute to information system research and practice as most of the 20 factors identified can be applicable to any information system project. This paper contributes to the implementation of ERP system, financial system, and CSFs research by identifying $20 \mathrm{CSF}$ derived from the literature. The significance of each identified factor for implementing financial ERP systems has been investigated in this study. In consonance with the objective of this study, it is apparent that factors presented are highly relevant to the implementation of financial ERP systems. The determination of CSFs based on the calculations of ADVIAN@ has revealed the contributions of the identified factors for successful implementation financial ERP systems. This study provides the management of finance with useful knowledge that can enable effective implementation strategies for supporting financial functions such as incorporating these factors into the planning, implementation and use of an ERP system that supports financial functions.

The limitations of this study are circumscribed by the availability of domain experts and underserved niche of ERP system implementation research that have delved deeply into the financial domain. However, most of the enterprise wide implementations of ERP systems are customized to the specific settings that make them potentially viable for studies on larger enterprises to present different findings. In addition, the results of expert evaluation, ranking and classification of factors may turn out differently. Nevertheless, the use of a scientific rigorous approach is highly desirable for this research because only a small amount of scientific research has been conducted in this subject area. Consequently, the chosen approach represents an appropriate method for obtaining a preliminary overview and substantiation of CSFs for implementing ERP systems to support financial functions in the higher education.

The direct implication of this study indicates a clear chasm in the literature on implementation of financial ERP systems. It is in the interest of management to observe very clearly the identified the challenges in the process of implementing ERP systems and to employ holistic strategies to mitigate the challenges. There exists a chasm between what has been investigated within the field of ERP system implementation, CSFs, and financial sector. The chasm can be exploited further by researchers and practitioners to better understand what makes a successful implementation of ERP systems for supporting financial functions. Furthermore, regardless of the study grounded on the settings of HEIs, the outcome can very well be generalized to other similar organizations whose financial management are in dare need of reformation and is of importance. Since many HEIs in developing countries such as South Africa have implemented financial ERP systems it could be contended that the outcome of this study can be generalized geographically. Hence, a possible action for future research endeavor in the domain of ERP system.

\section{ACKNOWLEDGMENT}

The first author would like to thank her fellow researchers for their inspirations and the Durban University of Technology for the support provided during the study.

\section{REFERENCES}

[1] A. Rabaa'i, "Identifying critical success factors of ERP Systems at the higher education sector," 2009.

[2] A. I. ALdayel, M. S. Aldayel, and A. S. Al-Mudimigh, "The critical success factors of ERP implementation in higher education in Saudi Arabia: a case study," Journal of Information Technology and Economic Development, vol. 2, no. 2, pp. 1, 2011.

[3] G. Tortorella, and C. E. Fries, "Reasons for adopting an ERP system in a public university in Southern Brazil."

[4] M. A. Al-Hadi, and N. A. Al-Shaibany, "Critical success factors (CSFs) of ERP in higher education institutions," International Journal, vol. 7, no. 4, pp. 92-95, 2017.

[5] R. C. Thompson, O. O. Olugbara, and A. Singh, "Deriving critical success factors for implementation of enterprise resource planning systems in higher education institution," African Journal of Information Systems, vol. 10, no. 1, 2018.

[6] I. K. Sowan, R. Tahboub, and F. Khamayseh, "University ERP Preparation Analysis: A PPU Case Study," International Journal of Advanced Computer Science And Applications, vol. 8, no. 11, pp. 345$352,2017$.

[7] J. Ram, D. Corkindale, and M.-L. Wu, "Implementation critical success factors (CSFs) for ERP: Do they contribute to implementation success and post-implementation performance?," International Journal of Production Economics, vol. 144, no. 1, pp. 157-174, 2013.

[8] O. O. Olugbara, B. M. Kalema, and R. M. Kekwaletswe, "Identifying critical success factors: the case of ERP systems in higher education," 2014. 
[9] M. Y. M. Al-Sabaawi, "Critical success factors for enterprise resource planning implementation success," International Journal of Advances in Engineering \& Technology, vol. 8, no. 4, pp. 496, 2015.

[10] A. Trigo, F. Belfo, and R. P. Estébanez, "Accounting information systems: The challenge of the real-time reporting," Procedia Technology, vol. 16, pp. 118-127, 2014.

[11] A. Y. Noaman, and F. F. Ahmed, "ERP systems functionalities in higher education," Procedia Computer Science, vol. 65, pp. 385-395, 2015.

[12] A. Abugabah, and L. Sanzogni, "Enterprise resource planning (ERP) system in higher education: A literature review and implications," International Journal of Human and Social Sciences, vol. 5, no. 6, pp. 395-399, 2010.

[13] J. Loonam, V. Kumar, A. Mitra, and A. Abd Razak, "Critical success factors for the implementation of enterprise systems: A literature review," Strategic Change, vol. 27, no. 3, pp. 185-194, 2018.

[14] A. S. Shatat, "Critical success factors in enterprise resource planning (ERP) system implementation: An exploratory study in Oman," Electronic Journal of Information Systems Evaluation, vol. 18, no. 1, pp. 36-45, 2015.

[15] M. Soliman, and N. Karia, "Antecedents for the Success of the Adoption of Organizational ERP Among Higher Education Institutions and Competitive Advantage in Egypt," Engineering, Technology \& Applied Science Research, vol. 7, no. 3, pp. 1719-1724, 2017.

[16] A. Epizitone, and O. O. Olugbara, "Critical success factors for ERP system implementation to support financial functions.," Academy of Accounting and Financial Studies Journal vol. 23, Issue 6, 2019.

[17] V. Linss, and A. Fried, "Advanced Impact Analysis: the ADVIAN ${ }^{\circledR}$ method-an enhanced approach for the analysis of impact strengths with the consideration of indirect relations," 2009.

[18] V. Linss, and A. Fried, "The ADVIAN ${ }^{\circledR}$ classification-A new classification approach for the rating of impact factors," Technological Forecasting and Social Change, vol. 77, no. 1, pp. 110-119, 2010.

[19] B. Guertler, and S. Spinler, "Supply risk interrelationships and the derivation of key supply risk indicators," Technological Forecasting and Social Change, vol. 92, pp. 224-236, 2015.

[20] A. Fried, "Performance measurement systems and their relation to strategic learning: A case study in a software-developing organization," Critical Perspectives on Accounting, vol. 21, no. 2, pp. 118-133, 2010.

[21] V. A. Bañuls, M. Turoff, and S. R. Hiltz, "Collaborative scenario modeling in emergency management through cross-impact," Technological Forecasting and Social Change, vol. 80, no. 9, pp. 17561774, 2013.

[22] J. L. Worrell, P. M. Di Gangi, and A. A. Bush, "Exploring the use of the Delphi method in accounting information systems research," International Journal of Accounting Information Systems, vol. 14, no. 3, pp. 193-208, 2013.

[23] F. Campos Fernandes Leandro, M. P. Mexas, and G. M. Drumond, "Identifying critical success factors for the implementation of enterprise resource planning systems in public educational institutions," Brazilian Journal of Operations \& Production Management, vol. 14, no. 4, pp. 529-541, 12, 2017.

[24] V. Arnold, "The changing technological environment and the future of behavioural research in accounting," Accounting \& Finance, vol. 58, no. 2, pp. 315-339, 2018.

[25] H. Abdelghaffar, and R. H. A. Azim, "Significant factors influencing ERP implementation in large organizations: Evidence from Egypt." pp. 1-16.

[26] C. Leyh, "Critical success factors for ERP projects in small and medium-sized enterprises-The perspective of selected German SMEs." pp. 1181-1190.

[27] C. Leyh, "Critical success factors for ERP projects in small and medium-sized enterprises - the perspective of selected ERP system vendors," Multidimensional Views on Enterprise Information Systems, pp. 7-22: Springer, 2016.

[28] S. Dezdar, and S. Ainin, "The influence of organizational factors on successful ERP implementation," Management Decision, vol. 49, no. 6, pp. 911-926, 2011.
[29] F. Belfo, and A. Trigo, "Accounting information systems: Tradition and future directions," Procedia Technology, vol. 9, pp. 536-546, 2013.

[30] M. A. AlSudairi, "Analysis and exploration of critical success factors of ERP implementation: a brief review," International Journal of Computer Applications, vol. 69, no. 8, 2013.

[31] T. A. Sykes, V. Venkatesh, and J. L. Johnson, "Enterprise system implementation and employee job performance: Understanding the role of advice networks," Mis Quarterly, vol. 38, no. 1, 2014.

[32] T. M. Somers, and K. G. Nelson, "A taxonomy of players and activities across the ERP project life cycle," Information \& Management, vol. 41, no. 3, pp. 257-278, 2004.

[33] F. Fui-Hoon Nah, J. Lee-Shang Lau, and J. Kuang, "Critical factors for successful implementation of enterprise systems," Business process management journal, vol. 7, no. 3, pp. 285-296, 2001.

[34] S. Finney, and M. Corbett, "ERP implementation: a compilation and analysis of critical success factors," Business Process Management Journal, vol. 13, no. 3, pp. 329-347, 2007.

[35] Y. B. Moon, "Enterprise Resource Planning (ERP): a review of the literature," International journal of management and enterprise development, vol. 4, no. 3, pp. 235-264, 2007.

[36] S. Dezdar, and A. Sulaiman, "Successful enterprise resource planning implementation: taxonomy of critical factors," Industrial Management \& Data Systems, vol. 109, no. 8, pp. 1037-1052, 2009.

[37] L. Shaul, and D. Tauber, "Critical success factors in enterprise resource planning systems: Review of the last decade," ACM Computing Surveys (CSUR), vol. 45, no. 4, pp. 55, 2013.

[38] R. G. Saade, and H. Nijher, "Critical success factors in enterprise resource planning implementation: a review of case studies," Journal of Enterprise Information Management, vol. 29, no. 1, pp. 72-96, 2016.

[39] K. Al-Fawaz, T. Eldabi, and A. Naseer, "Challenges and influential factors in ERP adoption and implementation," 2010.

[40] Z. Alias, E. Zawawi, K. Yusof, and N. Aris, "Determining critical success factors of project management practice: A conceptual framework," Procedia-Social and Behavioral Sciences, vol. 153, pp. 6169, 2014.

[41] M. Al-Mashari, A. Al-Mudimigh, and M. Zairi, "Enterprise resource planning: A taxonomy of critical factors," European Journal of Operational Research, vol. 146, pp. 352-364, 2003.

[42] E. Reitsma, and P. Hilletofth, "Critical success factors for ERP system implementation: a user perspective," European Business Review, vol. 30, no. 3, pp. 285-310, 2018.

[43] E. Ziemba, and I. Oblak, "Critical success factors for ERP systems implementation in public administration." pp. 1-19.

[44] H. Akkermans, and K. van Helden, "Vicious and virtuous cycles in ERP implementation: a case study of interrelations between critical success factors," European journal of information systems, vol. 11, no. 1, pp. 3546, 2002.

[45] S. Venkatraman, and K. Fahd, "Challenges and success factors of ERP systems in Australian SMEs," Systems, vol. 4, no. 2, pp. 20, 2016.

[46] A. Y. Aremu, A. Shahzad, and S. Hassan, "Determinants of Enterprise Resource Planning Adoption on Organizations' Performance Among Medium Enterprises," LogForum, vol. 14, no. 2, 2018.

[47] N. Ahmad, A. Haleem, and A. A. Syed, "Compilation of critical success factors in implementation of enterprise systems: a study on Indian organisations," Global journal of flexible systems management, vol. 13, no. 4, pp. 217-232, 2012.

[48] M. Ashja, A. H. Moghadam, and H. Bidram, "Comparative study of large information systems' CSFs during their life cycle," Information Systems Frontiers, vol. 17, no. 3, pp. 619-628, 2015.

[49] O. Françoise, M. Bourgault, and R. Pellerin, "ERP implementation through critical success factors' management," Business Process Management Journal, vol. 15, no. 3, pp. 371-394, 2009.

[50] P. Hanafizadeh, R. Gholami, S. Dadbin, and N. Standage, "The core critical success factors in implementation of enterprise resource planning systems," International Journal of Enterprise Information Systems (IJEIS), vol. 6, no. 2, pp. 82-111, 2010. 
[51] E. W. Ngai, C. C. Law, and F. K. Wat, "Examining the critical success factors in the adoption of enterprise resource planning," Computers in industry, vol. 59, no. 6, pp. 548-564, 2008.

[52] J. Ram, M.-L. Wu, and R. Tagg, "Competitive advantage from ERP projects: Examining the role of key implementation drivers," International Journal of Project Management, vol. 32, no. 4, pp. 663675, 2014.

[53] J. M. Denolf, J. H. Trienekens, P. N. Wognum, J. G. van der Vorst, and S. O. Omta, "Towards a framework of critical success factors for implementing supply chain information systems," Computers in industry, vol. 68, pp. 16-26, 2015.

[54] P. Saa, O. Moscoso-Zea, A. C. Costales, and S. Luján-Mora, "Data security issues in cloud-based Software-as-a-Service ERP." pp. 1-7.

[55] D. Saxena, and J. McDonagh, "Yet Another 'List'of Critical Success 'Factors' for Enterprise Systems: Review of Empirical Evidence and Suggested Research Directions." 